\title{
Multidisciplinary Approach in Full Mouth Rehabilitation - From Ruins to Riches in Oral Health
}

\author{
Dr. Prafulla Thumati ${ }^{1}$ M.D.S., Ph.D, Dr. K. Raghavendra Reddy ${ }^{2}$ M.D.S, \\ Dr. S. Padmaja ${ }^{3}$ M.D.S \\ Professor and HOD, Department of Prosthodontics, Dayananda Sagar College Of Dental Sciences, \\ Bangalore, Karnataka. India-560078. \\ Senior Lecturer, Department of Prosthodontics, Sri Sai College of Dental Surgery, Vikarabad, Andhra \\ Pradesh. India-501101. \\ Reader, Department of Prosthodontics, Dayananda Sagar College Of Dental Sciences, Bangalore, Karnataka. \\ India-560078
}

\begin{abstract}
Management of patients with a badly mutilated dentition is a complex situation for treating and a challenge to dentist's skills. The mutilated dentition due to caries, tilted, drifted and rotated teeth results in a compromised appearance, alteration in phonetics and difficulty in mastication. This article presents a multidisciplinary approach in rehabilitating mutilated dentition with ceramo polymer crowns (Ceramage), flexible partial denture and fiber splinting of natural pontic along with porcelain fused to metal work; to provide an ordered pattern of occlusal contact and articulation to optimize oral function.
\end{abstract}

Key words : ceramage. fiber splinting, fiber optic posts, mutilated dentition, natural pontic

\section{Introduction}

Esthetics has become a chief priority for patients today. The advertisings have led to almost a large percentage of patients refusing metallic restorations in their mouth and demand for restorations called white fillings. Planning and executing the restorative rehabilitation of a decimated occlusion is probably one of the most intellectually and technically demanding tasks facing a restorative dentist [1]. Occlusal rehabilitation therefore involves restoring the dentate or partially dentate mouth. The aim is to provide an ordered pattern of occlusal contact and articulation which will optimize oral function, occlusal stability and aesthetics. Patient's needs and demand vary much and should be individually assessed.

\section{A Case Report}

A 62 year male patient approached our private practice with a chief complaint of pain in the teeth and inability to chew food. The patient's medical history did not reveal any contraindications for the dental treatment. The clinical and radiographic examination, revealed the following findings:

Maxillary - Extensively decayed teeth in relation to 21,22,23; root stumps present in relation to 13,18 (Fig.1\&2). Mandibular - Periodontally compromised mandibular central and lateral incisors, root stumps in relation to 44 and 46.

2.1 Treatment protocol: A thorough oral prophylaxis was done followed by removal of carious lesions to determine the extent of tooth structure destroyed and the type of restoration to be placed. Periodontally compromised mandibular central and lateral incisors were kept under observation in dilemma either to retain or to extract the same. The treatment protocol was explained to the patient before the commencement of treatment.

\subsection{Maxillary dentition:}

Extraction of infected root stumps and severely mobile teeth was done in relation to 15 and recalled after a weak for suture removal. Root canal treatment was carried out in relation to 13,21,22 an 23 followed by fiber optic (Coltene Whaledent) post and core build up (Multicore Flow, Ivoclar Vivadent, Germany) (Fig.3\&4). Tooth preparation was done in relation to 11,12,13,14,21,22,23,24 and 25 (Fig.5) proceeded with soft tissue management (gingival retraction) and final impression using putty wash poly vinyl siloxane impression (Aquasil, Dentsply) along with face bow record and interocclusal registration.

The prepared teeth were provisionalised using hard acrylic resin temporary crown material (Systemp C \& B II, Ivoclar Vivadent, Germany) \& luted with Eugenol free zinc oxide cement (Fig.6). Indirect ceramopolymer (Ceramage, Shofu Inc, Japan) individual restorations were fabricated for the prepared teeth. These restorations were bonded by using Flowable composites [2] and Bonding agents (Beautifil Flow Plus and Beautifil bond Shofu Inc, Japan). The edentulous area 15,16,17 and 26 was replaced with Flexible partial denture (Fig. $7 \& 8$ ). 


\subsection{Mandibular dentition:}

Tooth preparation was done in relation to $33,37,43,45$ and 47 (Fig.9), proceeded with soft tissue management and final impression using putty wash poly vinyl siloxane impression. The PFM (Porcelain-fused to metal) crowns were cemented with Glass Ionomer Cement (Ketac Cem-3M ESPE) in relation to 33 to 37 and 43 to 47 (Fig.5,10\&11). After an observation period of 3 weeks, there was an improved prognosis in relation to 31,32 and 41 but 42 showed a poor prognosis with increased mobility. So, it was decided to extract 42 and the same tooth was used as a natural pontic to maintain esthetics and it was splinted to the adjacent natural teeth using fiber splint (Fig.12,13\& 14) after acid etching the enamel [3,4], then soaking the fiber-splint in bonding agent (resin adhesive) and light curing it on the tooth.

Oral hygiene instructions were given. The patient was followed up for four years. The patient is pleased with esthetics, function and comfort of the prostheses.

\section{Discussion}

Dentistry has long been a skilled craft, but now there is much more than that. Reorganized approach in full mouth rehabilitations should restore the structural and functional integrity of the dental arches that are compromised by decayed, missing, broken teeth. It should be more intellectually and technically planned and executed to optimize oral function, occlusal stability and esthetics. A case has to be treated not only by correcting worn out, broken or discolored teeth but also require optimizing oral function $[5,6]$.

The choice of restorative material used for treating this case was Ceramage to conserve the remaining tooth structure [7]. Ceramage is a light cured, zirconium silicate used for both anterior and posterior teeth due to its very high compressive and tensile strength, excellent color stability, outstanding wear resistance. It exhibits the same light transmission as natural teeth and has remarkable translucency. After the extraction of root stumps, it was decided to give a removable partial denture [8] to this patient as there was no posterior abutment. The Valplast flexible partial denture was preferred due to its ability to adapt to the constant movement and flexibility in the mouth.

To enhance patient's self confidence, patient's natural tooth was used as a pontic [9], and using woven fiber glass strands; splinting was done along with periodontal care like oral prophylaxis to preserve the soft and hard tissue. The fiber splint is esthetic and being translucent it is almost invisible.

\section{FIGURES}

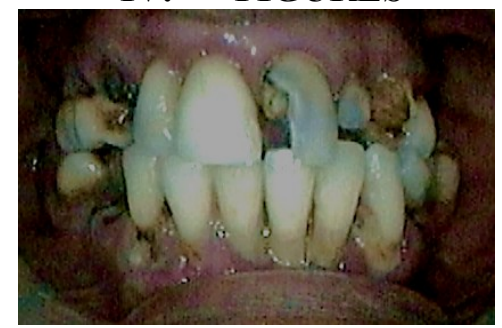

Fig. 1: intra oral view of dentition before treatment

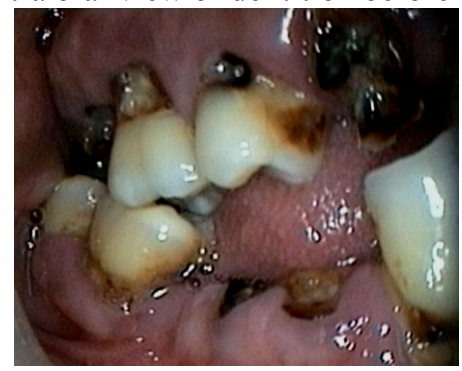

Fig. 2: root stumps

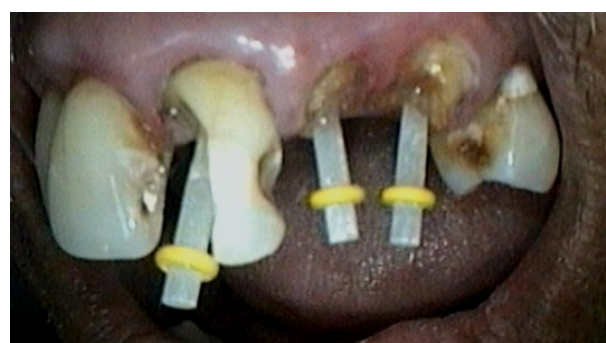

Fig. 3: fibre optic post for post $\&$ core build up 


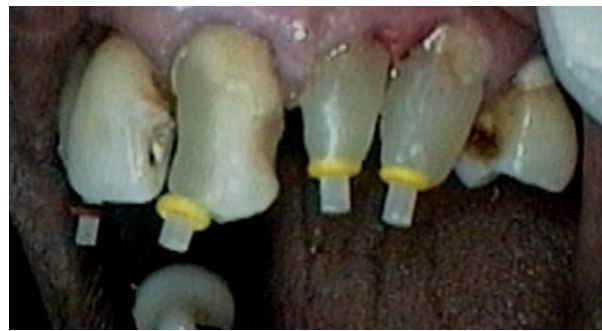

Fig. 4: cores build up done

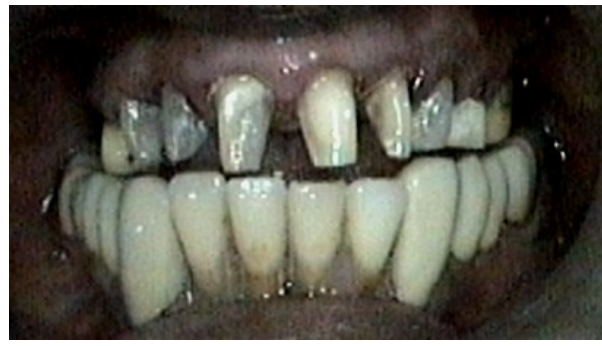

Fig. 5: tooth preparation done

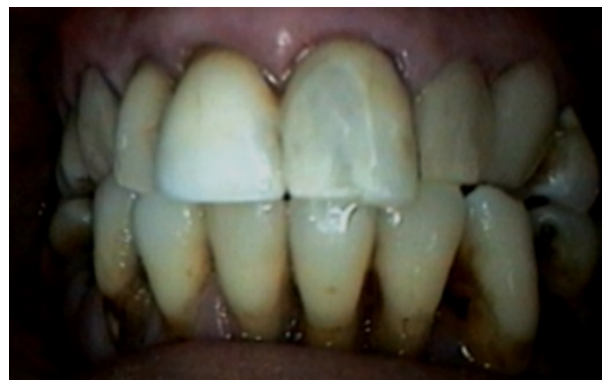

Fig. 6: prepared tooth provisionalised with heat polymerized hard acrylic resin

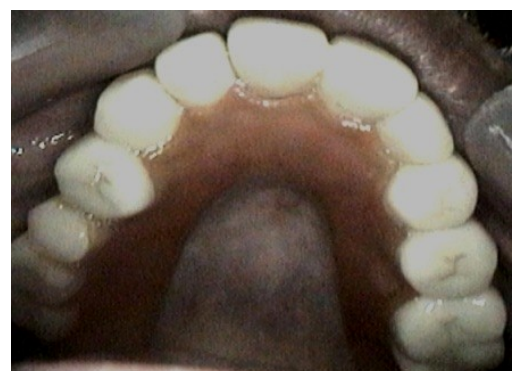

Fig. 7: flexible partial denture inserted

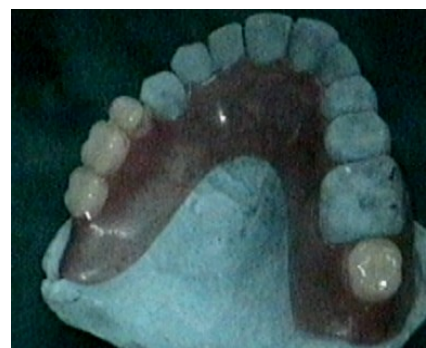

Fig. 8: flexible partial denture on the model 


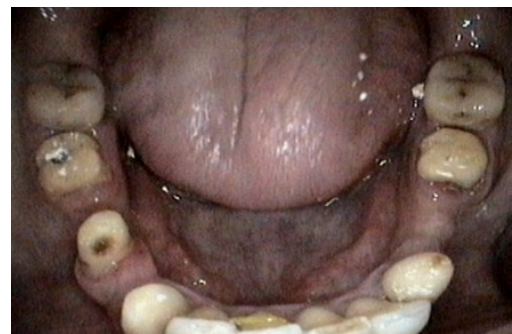

Fig. 9: tooth preparation done for abutment teeth

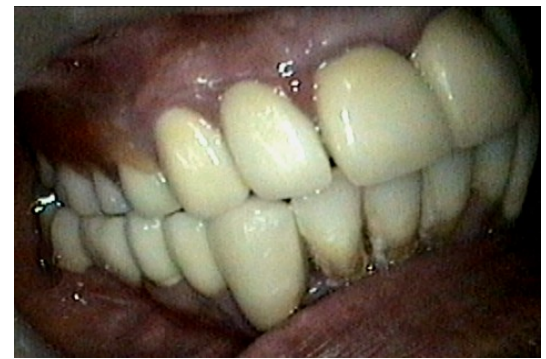

Fig. 10: ceramage individual crowns cemented with resin cement

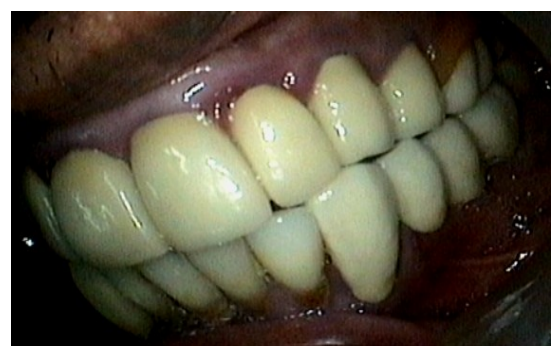

Fig. 11: ceramage individual crowns cemented with resin cement \& PFM with GIC

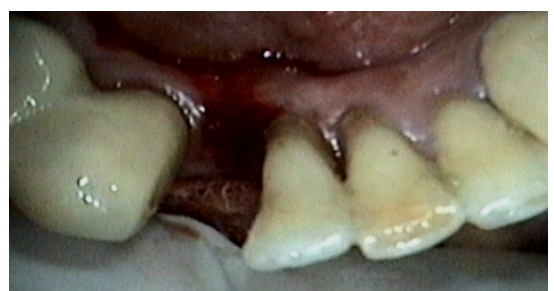

Fig. 12: tooth with bad prognosis extracted

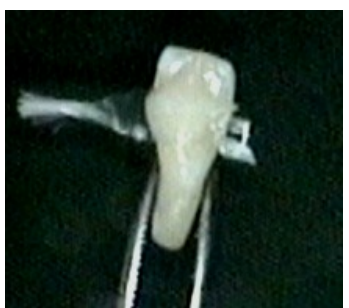

Fig. 13: tooth prepared for splinting

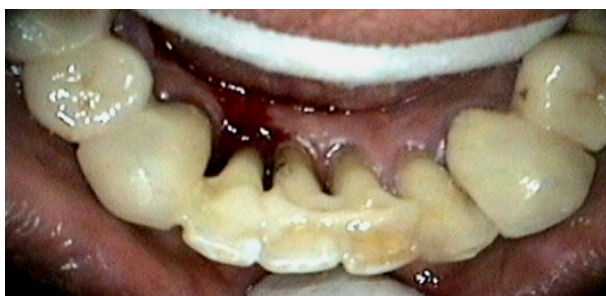

Fig. 14: natural teeth splinted using Fibre splint 


\section{Conclusion}

The reasons for undertaking occlusal rehabilitation may include the restoration of mutilated teeth. Increasingly, occlusal rehabilitation is also required to replace improperly designed and executed crown and bridgework. Full mouth rehabilitation involves restoring the teeth, jaw muscles and self-esteem back to a natural looking condition. Optimum oral health should be the prime objective of all rehabilitation procedures because the ultimate goal will always be to restore the mouth to health and function and preserve this status throughout the life of the patient.

\section{References}

[1] DZ. Irad and AP. Francis, Full mouth reconstruction fixed removable, Dent Clin North Am, 1987, 31-35.

[2] JM. Han, H. Lin, G. Zheng, A. Shinya, H. Gomi, J. Lin, Effect of nano filler on wear resistance and surface roughness of resin composites, Chin J Dent Res 15(1), 2012, 41-7.

[3] DN. Rudo, VM. Karbhari, Physical behaviors of fiber reinforcement as applied to tooth stabilization, Dent Clin North Am, 43, 1999, 7-35.

[4] HE. Strassler, A. Haeri, J. Gultz, New generation bonded reinforcing materials for anterior periodontal tooth stabilization and splinting, Dent Clin North Am, 43, 1999, 105-126.

[5] TK. Binkley, CJ. Binkley, A practical approach to full mouth rehabilitation, J Prosthet Dent, 57(3), 1987, $261-266$.

[6] Peter E.Dawson, Evaluation, diagnosis and treatment of occlusal problems (2nd Ed, Cv Mosby Company, St. Louis Baltimore, Toronto, 1989, 274-297)

[7] SK. Garoushi, A. Shinya, PK. Vallittu, Fiber-reinforced onlay composite resin restoration: A case report, J Contemp Dent Pract, 10(4), 2009, 104-10.

[8] J. Abduol, An innovative prostheses design for rehabilitation of severely mutilated dentition: A Case Report, J Adv Prosthodont 3, $2011,37-42$.

[9] GTR. Lee, Utilization of a natural tooth in acid-etch bridging, J Dent Child, 55, 1988, 201-204. 